\title{
Absorption enhancement of molecules in the weak plasmon-exciton coupling regime
}

\author{
Sinan Balci, ${ }^{1}$ Ertugrul Karademir, ${ }^{2}$ Coskun Kocabas, ${ }^{2}$ and Atilla Aydinli ${ }^{2, *}$ \\ ${ }^{1}$ Department of Astronautical Engineering, University of Turkish Aeronautical Association, 06790 Ankara, Turkey \\ ${ }^{2}$ Department of Physics, Bilkent University, 06800 Ankara, Turkey \\ ${ }^{*}$ Corresponding author: aydinli@fen.bilkent.edu.tr
}

Received July 4, 2014; revised July 20, 2014; accepted July 20, 2014;

posted July 21, 2014 (Doc. ID 216389); published August 18, 2014

\begin{abstract}
We report on the experimental and theoretical investigations of enhancing the optical absorption of organic molecules in the weak plasmon-exciton coupling regime. A metal-organic hybrid structure consisting of dye molecules embedded in the polymer matrix is placed in close vicinity to thin metal films. We have observed a transition from a weak coupling regime to a strong coupling one as the thickness of the metal layer increases. The results indicate that absorption of the self-assembled J-aggregate nanostructures can be increased in the weak plasmon-exciton coupling regime and strongly quenched in the strong coupling regime. A theoretical model based on the transfer-matrix method qualitatively confirms the experimental results obtained from polarization-dependent spectroscopic reflection measurements. (c) 2014 Optical Society of America

OCIS codes: (240.6680) Surface plasmons; (250.5403) Plasmonics; (300.1030) Absorption; (260.2160) Energy transfer. http://dx.doi.org/10.1364/OL.39.004994
\end{abstract}

Plasmonic nanostructures have been used for controlling light-matter interactions at nanoscale dimensions. Various promising applications in the areas of molecular imaging, biosensing, spectroscopy, solar cells, lasers, nonlinear optics, and optical communication have been emerging [1-5]. In particular, advances in the fabrication and chemical synthesis of nanomaterials in the fields of nanoscience and technology achieved in the last two decades have enabled the study of molecules whose excitonic modes interact with the nanoscale metallic structures supporting surface-plasmon-polariton (SPP) modes. SPPs are quantized collective oscillations of the conduction electrons, propagating along a metal and a dielectric interface and having decay lengths of about a few hundreds of nanometers in the dielectric and a few tens of nanometers in the metal layers. SPPs provide a tunable platform to study light-matter interaction at the nanoscale dimension and have been used to study subwavelength optics at the nanoscale because the incident electromagnetic field is focused and enhanced between the metal and the dielectric interface [6]. This giant field enhancement at the nanoscale level has been used in the fields of surface-enhanced Raman scattering, fluorescence enhancement, enhancement of absorption of the incident solar light in solar cells, and surfaceplasmon-enhanced light-emitting diodes [5-8]. Interactions between the metallic surfaces supporting SPPs and optical materials (e.g., organic and inorganic semiconductors) supporting excitonic modes have resulted in coupled quantum systems [9-17].

Coupling between the SPP modes and excitonic modes has been extensively studied in the strong coupling regime [9-17]. The strength of the interaction between the SP $\bar{P}$ and exciton modes is known to determine the final optical properties of the newly formed hybrid system. In the weak plasmon-exciton coupling regime, the inherent nature of SPP and exciton modes are not modified and thereby increase the absorption or emission rates of the molecules $[\underline{7}, \underline{8}]$. However, when the coupling is considered to be strong, SPP and exciton modes are modified by forming new coupled modes, and hence an anticrossing behavior has been observed in the dispersion curve [9-17]. Anticrossing is observed at the energy where both bare plasmon and bare exciton modes resonate at the same frequency. As it has been shown theoretically and experimentally, in the strong plasmon-exciton coupling regime, two newly formed energy states are separated by an energy value whose difference in energy is called the Rabi splitting energy and is given by

$$
\hbar \Omega_{R}=\left(4 V^{2}-\left(\gamma_{p}-\gamma_{e}\right)^{2}\right)^{1 / 2},
$$

where $V$ is the coupling parameter, and $\gamma_{p}$ and $\gamma_{e}$ are the linewidth (i.e., damping) of the bare plasmons and bare excitons, respectively.

Recent experiments have shown that the thickness of the metal films ( $\sim$ the skin depth of the metal) affects the properties of the system under study and thereby controls the amount of light absorbed or transmitted by the system [18-21]. For example, it has been shown that perforating a thick metal film by nanohole arrays greatly boosts the transmission of the incident light [6]. Conversely, when the thickness of the nanostructured metal film is decreased to around the skin depth of the metal ( $\sim 20 \mathrm{~nm})$, the opposite behavior has been observed, that is, transmission of the incident light is suppressed or, in other words, absorbance of the nanostructured film is increased [19]. In earlier studies, it has been observed that when the metal-film thickness is smaller than the penetration depth of the evanescent wave, absorbance of the metal film has been greatly increased [20,21]. In one of our earlier studies, we have shown that broadband critical coupling of the incident light can be achieved by adjusting the thickness of the metal film [18]. Recently, in a similar way, critical coupling has been investigated in coupled molecular and plasmonic cavity arrays [22]. Lately, tuning of plasmon-exciton coupling and hence the Rabi splitting energy in the strong-coupling regime by controlling the plasmon damping has been presented [15]. However, plasmon-exciton coupling below the skin 
depth of the metal has not been studied. Here, in this work, we investigate absorption enhancement of the molecules in the weak plasmon-exciton coupling regime. Strength of the plasmon-exciton coupling has been controlled, from the weak coupling regime (exhibiting no anticrossing behavior) to the strong coupling regime (exhibiting an anticrossing behavior), by plasmonic-layer thickness. In addition, concentration of the dye molecules is very critical and determines the transition point from the weak coupling regime to the strong coupling regime.

Figure 1(a) depicts the experimental setup used to measure the absorption enhancement of the molecules embedded in the polymer thin layer coated on an ultrathin metal film. A cyanine dye $\left[5,5^{\prime}, 6,6^{\prime}\right.$-tetrachloro-di(4-sulfobutyl)] benzimidazolocarbocyanine, TDBC, is used as the organic component because of (1) its strong dipole moment, (2) its ability to form self-assembled nanostructures of $J$ aggregates both in solution and in polymer matrices, and (3) its narrow absorption and emission linewidth [Figs. 1(b) and 1(c)]. Individual TDBC molecules self-assemble in a head-to-tail fashion to generate one-dimensional J-aggregate nanostructures. TDBC J aggregates have exciton resonance energy of $2.09 \mathrm{eV}$ $(590 \mathrm{~nm})$ because of the exciton-exciton coupling occurring between the individual dye molecules after the aggregation. The absorption linewidth of the J-aggregate excitons is very narrow $(\sim 51 \mathrm{meV}, \sim 16 \mathrm{~nm})$. The metalorganic hybrid structure has $\mathrm{J}$ aggregates supporting the excitonic mode as the organic contribution and $\mathrm{Ag}$ thin film support the plasmonic mode as the metal contribution. Polyvinylalcohol (PVA) powder is dissolved in

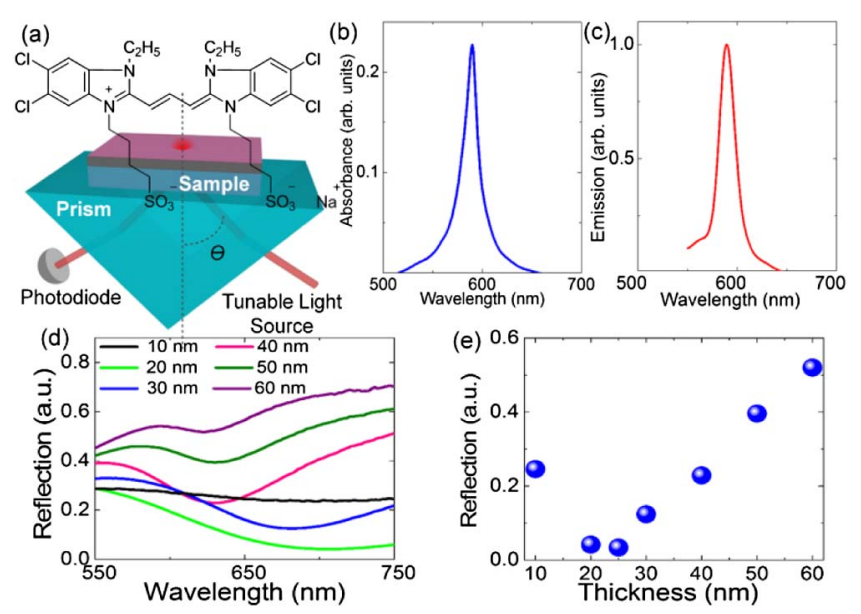

Fig. 1. (a) Schematic depiction of the Kretchsmann configuration (KC) used for excitation of surface plasmons on the Ag film by polarized incident laser light modulated with an acoustooptic tunable filter. Intensity of the reflected laser light is measured by a silicon photodiode. Incidence angle is varied with a motorized rotation stage with $0.1^{\circ}$ at each step. A plasmonicdispersion curve is generated by recording the reflection spectrum of the incident light at each $0.1^{\circ}$. The chemical structure of a TDBC molecule studied is shown in the inset. (b) Absorption spectrum of the $\mathrm{J}$ aggregates embedded in a PVA matrix. (c) Fluorescence emission spectrum from the same sample excited at $485 \mathrm{~nm}$ (Horiba Scientific FL-1057 TCSPC). (d) Reflection curves obtained from the bare Ag film with varying thickness at an incidence angle of $42.2^{\circ}$. (e) Using the reflection curves in (d), minima of the reflection have been drawn. boiling water to obtain a transparent water-based solution of the polymer. TDBC molecules are firstly dissolved in water and subsequently mixed with $1.5 \%$ PVA solution in a $1: 1(\mathrm{v} / \mathrm{v})$ ratio to obtain a $0.75 \%$ PVA and TDBC solution. Thin-film PVA ( $\sim 30 \mathrm{~nm})$ containing TDBC molecules is fabricated by spin-coating at $3000 \mathrm{rpm}$. Absorption and emission spectra of the resulting J-aggregate films are shown in Figs. 1(b) and 1(c), respectively. Both peaks are very narrow and very close to each other; the emission peak is only a few nanometers red-shifted with respect to the absorption peak.

The polariton-dispersion curves have been obtained by performing polarization-dependent spectroscopicreflection measurements in the Kretschmann configuration (KC) [Fig. 1(a)] by tilting the sample at an angle of $\theta$ and recording the reflectivity of the sample with the wavelength of the $p$-polarized incident light to excite surface plasmons at the metal-air interface. The glass sample containing the metal-organic hybrid structure is mounted on a BK-7 right-angle prism with an indexmatching liquid to preserve the optical continuity between the prism and the glass substrate. When the phase-matching condition is achieved, the recorded reflectivity in the photodiode goes to a minimum value as shown in Fig. 1(d) for the bare metal film with varying thicknesses. If the horizontal component of the momentum of the incident light $\left(k_{x}\right)$ matches the real part of the momentum of SPPs $\left(k_{\mathrm{spp}}\right)$, the excitation of the SPPs or SPP resonance condition is achieved. The dispersion relation of SPPs at a metal-dielectric interface can be defined as

$$
k_{x}=k_{0} n_{p} \sin (\theta)=k_{\mathrm{spp} 1}=\frac{2 \pi}{\lambda} \sqrt{\frac{\varepsilon_{m 1} \varepsilon_{d}}{\varepsilon_{m 1}+\varepsilon_{d}}},
$$

where $\lambda$ is the wavelength of the incident light, $k_{0}$ is the free space wavevector of the incident light, $\varepsilon_{m 1}$ and $\varepsilon_{d}$ are the dielectric constants of the metal and dielectric, respectively, $n_{p}$ is the refractive index of the prism, and $\theta$ is the SPP resonance angle. We can write the complex relative permittivity of the metal as $\varepsilon_{m}=\varepsilon_{m 1}+i \varepsilon_{m 2}$ and the complex SPP wave vector as $k_{\mathrm{spp}}=k_{\mathrm{spp} 1}+i k_{\mathrm{spp} 2}$. Then the imaginary wave vector of SPP controlling the internal damping (ohmic losses) is

$$
k_{\mathrm{spp} 2}=\frac{2 \pi}{\lambda} \frac{\varepsilon_{m 2}}{2\left(\varepsilon_{m 2}\right)^{2}}\left(\frac{\varepsilon_{m 1} \varepsilon_{d}}{\varepsilon_{m 1}+\varepsilon_{d}}\right)^{3 / 2} .
$$

The excitation of SPP on a flat metal surface in KC can be captured in the reflectivity spectrum with a Lorentzian line shape. The damping term determines the linewidth of the plasmon-resonance peak shown in Figs. 1(d) and $1(\mathrm{e})$. The coupling can be tuned by varying the plasmonic layer thickness and hence the damping of the SPPs [15]. As the thickness of the plasmonic layer increases, the lifetime of SPPs increases, indicating that the damping of the plasmonic mode decreases, which results in an increase in surface-plasmon-exciton interaction, hence large Rabi splitting energies. Here, we study the absorption enhancement of the molecules in the weak plasmon-exciton coupling regime where the polaritondispersion curve does not show an anticrossing behavior. 
By performing polarization-dependent spectroscopic reflection measurements of J-aggregate nanostructures embedded in the PVA matrix on Ag-coated surfaces of various thicknesses in $\mathrm{KC}$, we can probe the absorption enhancement of J aggregates. Figure 2(a) shows the evolution of the polariton-reflection curves as a function of $\mathrm{Ag}$ film thickness. The TDBC concentration $(1.3 \mathrm{mM})$ is kept constant in all the samples. Figure 2(b) displays the reflection curves for each plasmonic-layer thickness obtained from the dispersion curves shown in Fig. 2(a) at the incidence angle of $\sim 46^{\circ}$. The resonance at around $590 \mathrm{~nm}$ in the reflection curve indicates the absorbance of the self-assembled TDBC molecules. It is clear here that absorption of the $\mathrm{J}$ aggregates is enhanced with the increase in the Ag film thickness, as shown in Fig. 2(c). When the plasmonic-layer thickness reaches $\sim 30 \mathrm{~nm}$, an anticrossing behavior emerges at the energy corresponding to a measured absorption maximum of $\mathrm{J}$ aggregate as a sign of the strong coupling regime. As argued above, in this regime, new optical modes are formed in the polariton-dispersion curve. By increasing the plasmonic-layer thickness to higher values, the quality factor of the plasmon resonance increases [Figs. 1(d) and 1(e)], or damping of the plasmonic mode decreases, and thus the Rabi splitting energy widens in the dispersion curve due to the increase in the coupling strength. At the exciton resonance energy, in the weakcoupling regime, plasmonic field is greatly enhanced whereas in the strong coupling regime, the plasmonic field is strongly quenched.
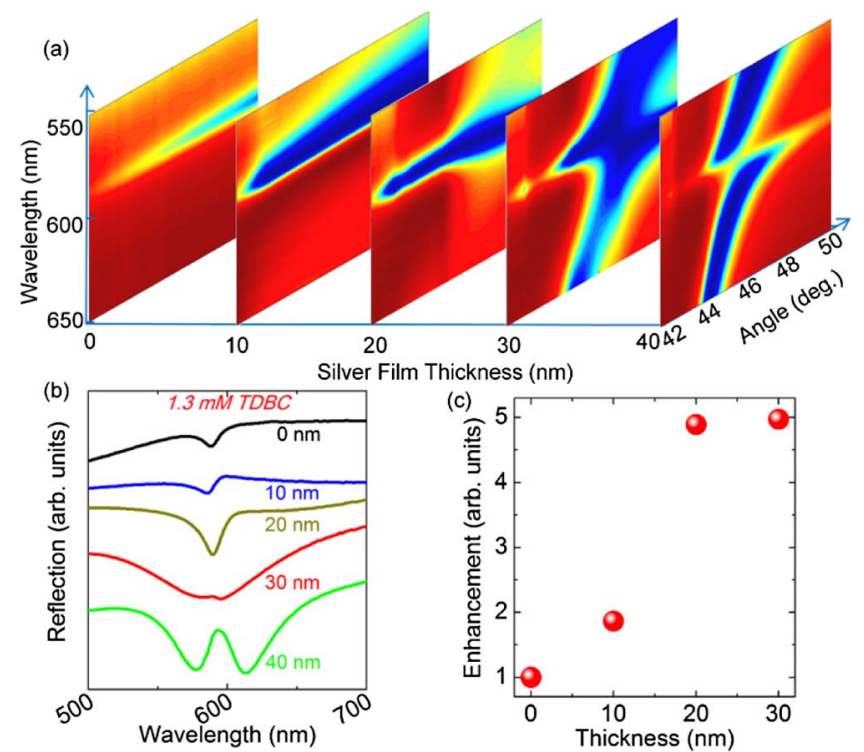

Fig. 2. Experimental data showing the absorption enhancement of the $J$ aggregates operating in the weak plasmon-exciton coupling regime. (a) Evolution of the polariton-reflection curves as a function of $\mathrm{Ag}$ film thickness. Concentration of TDBC is constant at $1.3 \mathrm{mM}$. (b) Evolution of the reflection curves as a function of the plasmonic-layer thickness. Transition from a weak to a strong coupling regime is observed at $\sim 30 \mathrm{~nm}$ thick Ag film. (c) Absorption of J aggregates is enhanced with the increase in the plasmonic layer thickness. For films thicker than $\sim 30 \mathrm{~nm}$, the coupling is in the strong coupling regime and the absorption of TDBC is strongly quenched and thus not included in the graph.
In order to theoretically calculate the polaritonreflection curves, optical constants $(n, k)$ of TDBC molecules as a function of wavelength for a specified TDBC concentration were experimentally measured using a variable-angle spectroscopic ellipsometer [15]. We calculated the dispersion relation of plasmon-exciton system by solving Maxwell's equations for each layer using the transfer-matrix method. Such a model with experimentally obtained optical constants of J-aggregate film and a plasmonic layer [23] provides a classical explanation of the plasmon-exciton coupling [15]. Figure 3(a) shows the theoretically calculated reflectivity maps for Jaggregate film fabricated from a $1.3 \mathrm{mM}$ TDBC in PVA solution coated onto the varying thickness of the Ag film. Figure 3(b) shows the reflection spectra for various thicknesses. The enhancement we calculate using a transfer-matrix method for $1.3 \mathrm{mM}$ TDBC is qualitatively in very good agreement with the experimental data shown in Fig. 2. Furthermore, the effect of the TDBC concentration on the absorption enhancement has been studied. Dispersion curves have been calculated for 0.6, 2.5, and 5.0 mM TDBC. Figure 3(c) shows the reflection spectra obtained from the dispersion curve of $5.0 \mathrm{mM}$ TDBC on various metal thicknesses. Absorption of the $\mathrm{J}$ aggregate is enhanced when the plasmonic-layer thickness increases, as shown in Fig. 3(d). Absorption enhancement of $\sim 15$ times for $0.6 \mathrm{mM}$ TDBC can be observed in the weak plasmon-exciton coupling regime. When the concentration of the TDBC dye in the PVA matrix has been decreased from 5.0 to $0.6 \mathrm{mM}$, it is very clear in the figure that the transition point from weak to strong coupling is shifted from 35 to $40 \mathrm{~nm} \mathrm{Ag}$ film thickness [Fig. $3(\mathrm{~d})$ ]. This is an expected result because,
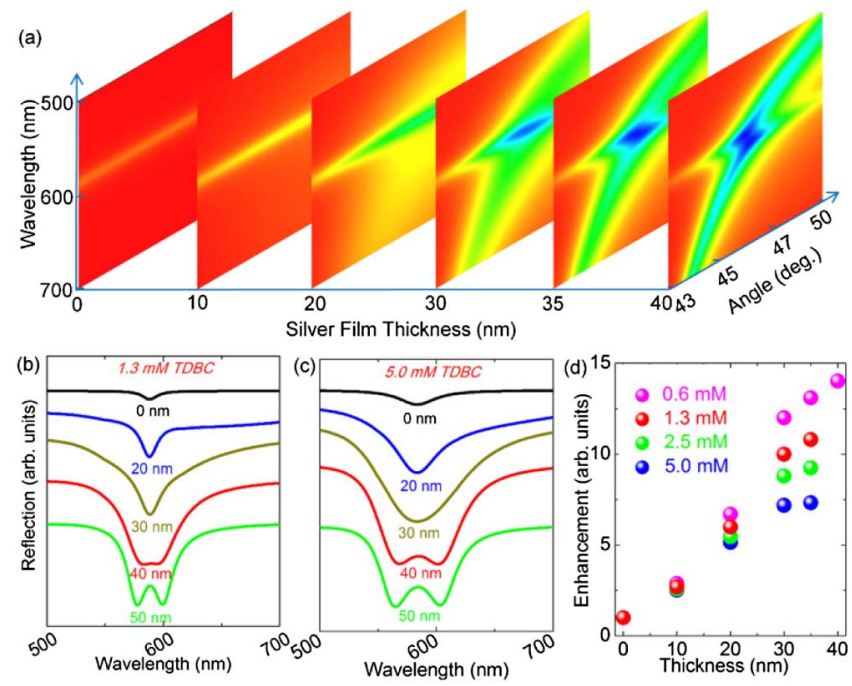

Fig. 3. (a) Calculated polariton-reflection curves for $1.3 \mathrm{mM}$ TDBC in the PVA matrix and with varying plasmonic-layer thicknesses. The blue and red regimes indicate the reflectivity minima and maxima, respectively. (b) The reflection spectra for $1.3 \mathrm{mM}$ TDBC for various metal film thicknesses obtained from the dispersion curves in (a). (c) The reflection spectra for $5.0 \mathrm{mM}$ TDBC for various metal-film thicknesses obtained from the calculated dispersion curves. (d) Calculated absorption enhancement of $\mathrm{J}$ aggregates as a function of metal-film thickness and J-aggregate concentration. The transition point from weak to strong coupling regime is shifted to the higher metal-film thickness values as the J-aggregate concentration decreases. 

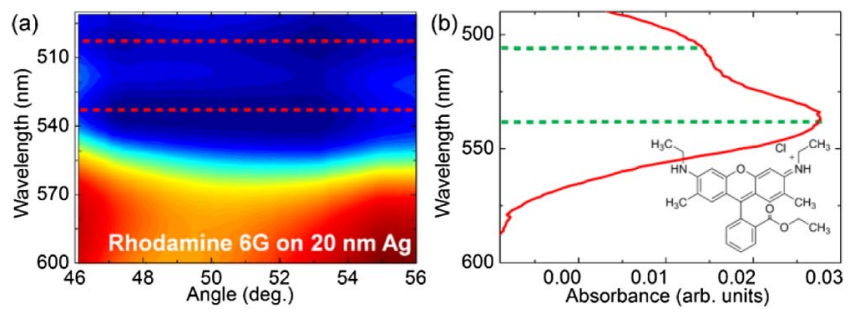

Fig. 4. Enhancing absorption of Rhodamine 6G molecules. (a) Polariton-reflection curve from $20 \mathrm{~nm}$ Ag film coated with the Rhodamine 6G molecules in the PVA matrix. (b) Absorption spectrum of the Rhodamine 6G molecules in the PVA matrix. The inset shows the chemical structure of a Rhodamine $6 \mathrm{G}$ molecule. The green dotted lines show the position of the excitontransition-energy levels in the absorption spectrum of the molecule.

in the strong-coupling regime, the coupling energy increases linearly with the square root of the number of dye molecules and, quality factor of the plasmonic mode increases with the commensurate increase in metal-film thickness. After decreasing the concentration of the dye molecules from 5 to $0.6 \mathrm{mM}$ to reach the strong coupling level, a higher-quality-factor plasmonic mode (thick Ag film) is needed, as shown Figs. 1(d) and 1(e). Even higher absorption enhancement of the dye molecules could be achieved by decreasing the concentration of the J aggregate to $\sim 0.01 \mathrm{mM}$, the lowest concentration of the TDBC dye in the polymer matrix where $\mathrm{J}$ aggregation can be observed. It is obvious in this case that transition from the weak to the strong coupling point (i.e., metal-film thickness) will be shifted to the higher metal-filmthickness values.

The absorption enhancement observed in the weak coupling regime has been applied in enhancing the absorption of a laser dye, Rhodamine 6G, having two separate excitonic broadbands (i.e., weak oscillator strength). Reflection of the incident light from the TDBC molecules is shown in Fig. 2(b), where the black and dark yellow curves indicate the reflection from the glass surface containing only TDBC molecules in the PVA matrix and TDBC molecules in a 30-nm-thick PVA film located on a 20-nm-thick Ag film. A polariton-reflection curve from the sample containing $20 \mathrm{~nm}$ Ag film coated with the TDBC molecules in the PVA matrix is demonstrated in Fig. 2(a). The result shows that the absorption of the aggregated TDBC molecules is enhanced and there is no anticrossing, that is, the indication of the weak plasmonexciton coupling, in the dispersion curve shown in Fig. 2(a) for $20 \mathrm{~nm}$. In a similar way, a polariton-reflection curve from the sample containing $20 \mathrm{~nm} \mathrm{Ag}$ film coated with the Rhodamine 6G molecules in the PVA matrix is demonstrated in Fig. 4(a). The dotted red lines indicate the exciton transition energy levels in the Rhodamine 6G molecule. The absorption spectrum of the dye in the PVA matrix is given in Fig. 4(b). The broad excitontransition-energy levels of Rhodamine $6 \mathrm{G}$ can be easily located and mapped out in the polariton-reflection curve, as shown in Fig. 4(a).

In conclusion, we have experimentally and theoretically studied the absorption enhancement of the dye molecules placed in close vicinity to metal films in the weak plasmon-exciton coupling regime. It is observed that absorption of the dye molecules is greatly enhanced in the weak-coupling regime and strongly quenched in the strong-coupling one. Transition from weak-coupling regime to strong-coupling regime has been observed at a critical plasmonic-layer thickness. The transition point depends on both the number of dye molecules (concentration) present in the polymer matrix and the metal-film thickness. The broad excitonic bands of the laser dye Rhodamine 6G molecules have been mapped out. The results open up ways for understanding energy transfer and coherent coupling in plasmonic devices with controlled coupling and optical properties at nanoscale dimensions. The results might further open new detection and analysis techniques of fluorophores used in biochemical and biophysical investigations by enhancing absorption of the fluorophores.

This work has been supported by grants 110T790, 110T589, and 112T091 from TUBITAK.

\section{References}

1. T. Yoshie, A. Scherer, J. Hendrickson, G. Khitrova, H. M. Gibbs, G. Rupper, C. Ell, O. B. Shchekin, and D. G. Deppe, Nature 432, 200 (2004).

2. D. G. Lidzey, D. D. C. Bradley, A. Armitage, S. Walker, and M. S. Skolnick, Science 288, 1620 (2000).

3. D. G. Lidzey, D. D. C. Bradley, T. Virgili, A. Armitage, M. S. Skolnick, and S. Walker, Phys. Rev. Lett. 82, 3316 (1999).

4. J. R. Tischler, M. S. Bradley, Q. Zhang, T. Atay, A. Nurmikko, and V. Bulovic, Org. Electron. 8, 94 (2007).

5. M. S. Tame, K. R. McEnegy, S. K. Ozdemir, J. Lee, S. A. Maier, and S. Kim, Nat. Phys. 9, 329 (2013).

6. W. L. Barnes, A. Dereux, and T. W. Ebbesen, Nature 424, 824 (2003).

7. F. Tam, G. P. Goodrich, B. R. Johnson, and N. J. Halas, Nano Lett. 7, 496 (2007).

8. J. Vuckovic, M. Loncar, and A. Scherer, IEEE J. Quantum Electron. 36, 1131 (2000).

9. G. A. Wurtz, P. R. Evans, W. Hendren, R. Atkinson, W. Dickson, R. J. Pollard, and A. V. Zayats, Nano Lett. 7 , 1297 (2007).

10. J. Dintinger, S. Klein, F. Bustos, W. L. Barnes, and T. W. Ebbesen, Phys. Rev. B 71, 035424 (2005).

11. T. K. Hakala, J. J. Toppari, A. Kuzyk, M. Pettersson, H. Tikkanen, H. Kunttu, and P. Torma, Phys. Rev. Lett. 103, 053602 (2009).

12. T. Schwartz, J. A. Hutchison, C. Genet, and T. W. Ebbesen, Phys. Rev. Lett. 106, 196405 (2011).

13. S. Balci, Opt. Lett. 38, 4498 (2013).

14. Y. B. Zheng, B. K. Juluri, L. L. Jensen, D. Ahmed, M. Lu, L. Jensen, and T. J. Huang, Adv. Mater. 22, 3603 (2010).

15. S. Balci, C. Kocabas, S. Ates, E. Karademir, O. Salihoglu, and A. Aydinli, Phys. Rev. B 86, 235402 (2012).

16. D. E. Gomez, K. C. Vernon, P. Mulvaney, and T. J. Davis, Nano Lett. 10, 274 (2010).

17. A. E. Schlather, N. Large, A. S. Urban, P. Nordlander, and N. J. Halas, Nano Lett. 13, 3281 (2013).

18. S. Balci, C. Kocabas, and A. Aydinli, Opt. Lett. 36, 2770 (2011).

19. J. Braun, B. Gompf, G. Kobiela, and M. Dressel, Phys. Rev. Lett. 103, 203901 (2009).

20. H. Kano and S. Kawata, Appl. Opt. 33, 5166 (1994).

21. A. Ikehata, X. Li, T. Itoh, and Y. Ozaki, Appl. Phys. Lett. 83 , 2232 (2003).

22. R. Adato, A. Atar, S. Erramilli, and H. Altug, Nano Lett. 13, 2584 (2013).

23. E. D. Palik, Handbook of Optical Constants of Solids (Academic, 1985). 